\title{
Article \\ A Plasmonic Infrared Multiple-Channel Filter Based on Gold Composite Nanocavities Metasurface
}

\author{
Jialin Zhang ${ }^{1}{ }^{(}$, Xuanyi Yu ${ }^{1}$, Jingxin Dong ${ }^{2}{ }^{\oplus}$, Weiji Yang ${ }^{1}$, Shuang Liu ${ }^{1}$, Chongyang Shen ${ }^{1}$, Jiacheng Duan ${ }^{1}$ \\ and Xiaoxu Deng ${ }^{1, *}$
}

check for

updates

Citation: Zhang, J.; Yu, X.; Dong, J.; Yang, W.; Liu, S.; Shen, C.; Duan, J.; Deng, X. A Plasmonic Infrared Multiple-Channel Filter Based on Gold Composite Nanocavities Metasurface. Nanomaterials 2021, 11, 1824. https://doi.org/10.3390/ nano11071824

Academic Editor: Andrey B. Evlyukhin

Received: 7 June 2021

Accepted: 12 July 2021

Published: 14 July 2021

Publisher's Note: MDPI stays neutral with regard to jurisdictional claims in published maps and institutional affiliations.

Copyright: (C) 2021 by the authors. Licensee MDPI, Basel, Switzerland. This article is an open access article distributed under the terms and conditions of the Creative Commons Attribution (CC BY) license (https:// creativecommons.org/licenses/by/ $4.0 /)$.
1 State Key Laboratory of Advanced Optical Communication Systems and Networks, Key Laboratory for Laser Plasmas (Ministry of Education), School of Physics and Astronomy, Shanghai Jiao Tong University, Shanghai 200240,China; dorismalfoy@sjtu.edu.cn (J.Z.); yxy1593725@sjtu.edu.cn (X.Y.); yangweiji@sjtu.edu.cn (W.Y.); 503313461@sjtu.edu.cn (S.L.); cy_shen@sjtu.edu.cn (C.S.); duanjiacheng@sjtu.edu.cn (J.D.)

2 School of Electronic Information and Electrical Engineering, Shanghai Jiao Tong University, Shanghai 200240, China; jx_dong@sjtu.edu.cn

* Correspondence: xxdeng@sjtu.edu.cn

\begin{abstract}
A plasmonic near-infrared multiple-channel filter is numerically and experimentally investigated based on a gold periodic composite nanocavities metasurface. By the interference among different excited plasmonic modes on the metasurface, the multipeak extraordinary optical transmission (EOT) phenomenon is induced and utilized to realize multiple-channel filtering. Investigated from the simulated transmission spectrum of the metasurface, the positions and intensity of transmission peaks are tuned by the geometrical parameters of the metasurface and environmental refractive index. The fabricated metasurface approached transmission peaks at $1128 \mathrm{~nm}, 1245 \mathrm{~nm}$, and $1362 \mathrm{~nm}$, functioning as a three-passbands filter. With advantages of brief single-layer fabrication and multi-frequency selectivity, the proposed plasmonic filter has potential possibilities of integration in nano-photonic switching, detecting and biological sensing systems.
\end{abstract}

Keywords: metasurface; nanocavities; plasmonic filter

\section{Introduction}

Metamaterial, especially its two-dimensional equivalents, i.e., metasurface, has aroused widespread attention due to its splendid electromagnetic wave manipulations properties, such as beam steering [1], radiation patterns reconfiguration [2], and nearfield transformation [3]. Plasmonic metamaterials based on metal nanocavities, exhibiting notable optical properties, including extraordinary optical transmission (EOT) [4], negative refractive index [5], and enhancement of nonlinear effect [6,7], has been an active research field in past decades, which provide great prospects of the application in sensing [8-10], plasmonic color filtering [11-14], and subdiffractive imaging [15], etc. Metallic nanocavity concentrates optical energy to deep subwavelength regions by the excitation of surface plasmons, inducing confinement of electromagnetic fields with frequency-selective features [16,17]. Periodic nanometallic cavities arrays in metamaterials support plasmons near-field coupling among cavities [18], leading to collective resonances such as the EOT phenomenon at selectable wavelength. Further on, the composite cavities structure is introduced into the metamaterial [19-21], which generates an interaction giving rise to plasmonic hybridization and strong optical field coupling among spectrally-overlapped modes, resulting in the additive spectral response of metamaterial [22-25]. For example, a polarization-insensitive NIR filter is presently based on asymmetry metallic elliptical and circle nanocavities array metasurface [26], exhibiting $79 \mathrm{~nm}$ narrow linewidth generated by a Fano resonance. The invertible plasmonic spin-Hall effect at the nanoscale is achieved by breaking the spin degeneracy through the interference among the different plasmon resonances in the U-shaped cavity metasurface [27]. An absorptive-type metasurface color filter 
is realized through truncated-cone hyperbolic metamaterial absorbers consisting of several layers of metal-dielectric films with tapered angles [28]. Hence, with increasingly matured nanofabrication, the realization of multi-wavelength and high-efficiency sub-wavelength optical manipulation with a single-layer metasurface is worth investigating.

In this paper, a multi-channel infrared plasmonic filter based on a gold periodic composite nanocavities metasurface is numerically and experimentally investigated. Plasmonic modes excited in single horizontal nanocavity structure and double vertical nanocavities structure are simulated by the finite-difference time-domain (FDTD) solutions, of which the interference induces multipeak extraordinary optical transmission phenomenon utilized in a plasmonic multi-frequency selective filter. By numerically analyzing the transmission spectrum of the metasurface, the transmission coefficient, operating channels, and linewidths of the plasmonic filter are tuned by cavities' geometric parameters and metasurface periods, especially the operating channels, which are also tuned by the environment refractive index. The maximum transmission intensity and narrowest FWHM (full width at the half-maximum) of the EOT peaks are optimized in the simulation as $73 \%$ and $8 \mathrm{~nm}$, respectively. The gold composite nanocavities metasurface was fabricated by ElectronBeam Lithography and Ion Beam Etching technique. The transmission spectrum of the fabricated metasurface was measured by a Fourier-transform infrared spectrometer with three passbands at wavelength $1128 \mathrm{~nm}, 1245 \mathrm{~nm}$, and $1362 \mathrm{~nm}$, which has good accordance in spectral positions with the simulation. Due to the advantages of multi-frequency selectivity and the single-layer simple fabrication process, the plasmonic filter based on the composite nanocavities metasurface has the potential to be a spatial component in high precision optical systems such as biosensors and photodetection technologies.

\section{Materials and Methods}

The schematic of a periodic composite nanocavities metasurface on $\mathrm{SiO}_{2}$ substrate is shown in Figure 1. The unit cell of the metasurface has composite gold nanocavities, including one horizontal cavity connected with two vertical cavities on the opposite side. The length of the horizontal cavity is $\mathrm{L} 1=800 \mathrm{~nm}$, and the lengths of vertical nanocavities are L2 $=200 \mathrm{~nm}$ and L3 $=300 \mathrm{~nm}$. Two vertical cavities are pointing upwards and downwards with a distance S1 $=200 \mathrm{~nm}$ and S2 $=100 \mathrm{~nm}$ to the left and right end of the horizontal cavity, respectively. The distance between two vertical cavities is $S=300 \mathrm{~nm}$. The width of all three composite nanocavities is $\mathrm{a}=100 \mathrm{~nm}$. The periods of the metasurface are $P x=1200 \mathrm{~nm}$ and $\mathrm{Py}=1100 \mathrm{~nm}$. The thickness of the metasurface is $\mathrm{h}=225 \mathrm{~nm}$. The environment refractive index $\mathrm{n}_{\mathrm{e}}=1$. The refractive index of $\mathrm{SiO}_{2}$ substrate is $n=1.45$. The transmission spectra and the electric field distributions of the metasurface are simulated by the three-dimensional FDTD solution. In the 3D FDTD simulations, the Y-polarized light $(900 \mathrm{~nm}-1500 \mathrm{~nm})$ is illuminated along the Z-axis. The dielectric parameter of Au in the FDTD simulation is set as the Palik model. A semi-infinite $\mathrm{SiO}_{2}$ substrate is added below the metasurface. Periodic boundary conditions (PBCs) are used in the $\mathrm{x}$ - and $\mathrm{y}$-directions, and perfectly matched layers (PMLs) are applied in the z-direction. The minimum mesh step is set as $0.25 \mathrm{~nm}$.

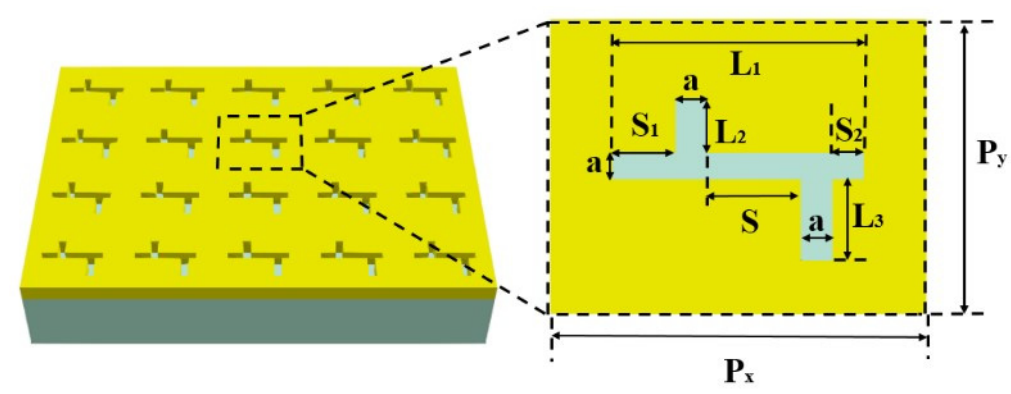

Figure 1. A schematic diagram of the periodic composite nanocavities metasurface. 
In the fabrication process, the $225 \mathrm{~nm}$-thick gold film was deposited on the $\mathrm{SiO}_{2}$ substrate by the Electron Beam evaporator (Denton Electron Beam Evaporator, Shanghai, China). The thickness of the $\mathrm{SiO}_{2}$ substrate is $1 \mathrm{~mm}$. The composite nanocavities structure was then fabricated on the gold film by Electron-beam lithography (EBL, Vistec EBPG-5200+, Shanghai, China) process with AR-P 6200 polymethylmethacrylate (PMMA) positive photoresist of $200 \mathrm{~nm}$ thickness. The structure was then developed and fixed using MIBK and IPA. Ion Beam Etching (IBE, Ion Beam Etching System, Shanghai, China) technique was utilized to remove the photoresist using Ar gas. The size of the fabricated area is $1.2 \mathrm{~mm} \times 1.1 \mathrm{~mm}$. The geometrical parameters were measured in the scanning electron microscopy (SEM, Zeiss Ultra Plus Field Emission Scanning Electron Microscope, Shanghai, China) image. The transmission spectrum of the fabricated metasurface is detected by a Fourier-transform infrared spectrometer (Fourier-transform infrared spectrometer, Shanghai, China) with a linear-polarized source.

\section{Results and Discussion}

\subsection{Simulated Results}

The transmission spectrum of the single-horizontal nanocavity metasurface, the double-vertical nanocavities metasurface, and the composite nanocavities metasurface are simulated by the FDTD solution. Under a Y-polarized incident field, the single-horizontal cavity metasurface obtains an EOT peak of $82 \%$ at $1132 \mathrm{~nm}$, shown as the green curve in Figure 2. At the $1132 \mathrm{~nm}$ transmission peak, the electric field is enhanced within the horizontal cavity, as shown in Figure 3a, which is induced by the excited surface plasmon polaritons (SPPs). Consequently, the single-horizontal cavity structure serves as the basic mode structure. The simulated transmission spectrum (blue curve in Figure 2) of doublevertical cavities metasurface under the $X$-polarization incident has two transmission peaks at wavelengths $1205 \mathrm{~nm}$ and $1280 \mathrm{~nm}$ of $23 \%$ and $92 \%$ transmittance, respectively, which has no transmission peak at this waveband with Y-polarization incident light. Similarly, the electric field is confined within the left and right vertical cavity at transmission peaks, respectively, as shown in Figure 3b. The transmission spectrum of composite cavities metasurface with horizontal and vertical cavities under Y-polarized incident is simulated, shown as the red curve in Figure 2, in which three EOT peaks, namely peak I, II, and III, appear at $1126 \mathrm{~nm}, 1255 \mathrm{~nm}$, and $1350 \mathrm{~nm}$ with $73 \%, 36 \%$, and $43 \%$ transmittances, respectively. At transmission peak I, the electric field is enhanced within the horizontal cavity, shown in Figure 3c, which is directly motived by the basic mode before. Meanwhile, newly generated transmission peaks II and III induced from the interaction between different modes, where the electric field is enhanced within the L-shaped junctions of the vertical cavities and horizontal cavity, which are red-shifted in peak position comparing with the plasmonic modes in vertical cavities. Plasmonic hybridization is generated in composite nanocavities, resulting in additive transmission spectral response. At three transmission peaks, the near-field electrical field is confined within the nanocavities due to the SPPs resonance, as shown in Figure 4. The confinement of the near-field electrical field is also influenced by the surrounding environment [29]. The multipeak extraordinary optical transmission phenomenon implemented by the composite nanocavities metasurface is utilized in multi-channel infrared plasmonic filtering.

To meet various practical demands, the impact on the transmission spectra with several geometrical parameters of the metasurface is investigated, offering different parameter selection strategies of choosing transmission intensity, frequency positions and spacing of transmission peaks together or individually. The influence of Au film thickness on the transmission spectrum of composite nanocavities metasurface is investigated by FDTD. With Au film thickness h increasing from 200 to $400 \mathrm{~nm}$, EOT peak I presents an obvious red shift and a higher transmittance with the broadening of FWHM, and Peak II and III are just slightly blue-shifted in Figure 5, which leads to peak I and peak II overlapped when $h$ is over $350 \mathrm{~nm}$. The increase in Au thickness causes more electric fields confined inside the horizontal cavity and more material dissipation, leading to the variation of transmission 
peak I both in FWHM and amplitude. Peaks II and III are attributed to the coupling between different plasmonic modes, of which resonance wavelengths are almost unaffected by the increase in $\mathrm{h}$. Therefore, EOT peak positions and intensity can be simultaneously selected by adjusting the thickness of Au film.

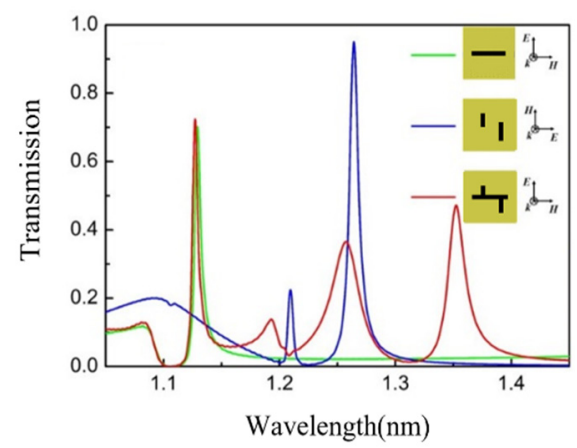

Figure 2. Normalized transmission spectrum (T) of the single nanocavity metasurface, double nanocavities metasurface, and composite nanocavities metasurface.

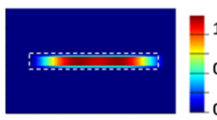

(a)

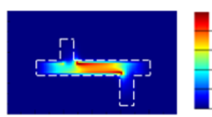

peak I

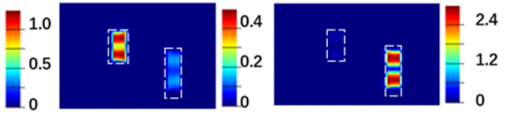

(b)

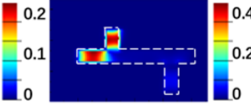

peak II

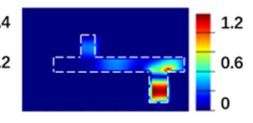

peak III

(c)

Figure 3. Simulated electric field distributions at the corresponding transmission peaks in crosssections of (a) the single-horizontal nanocavity structure, (b) the double-vertical nanocavities structure, and (c) the composite nanocavities structure.

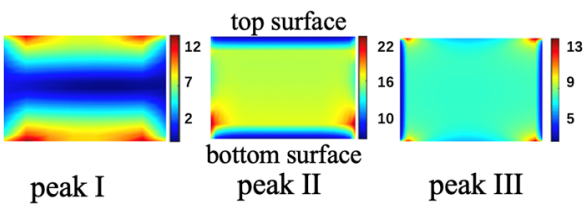

Figure 4. Simulated electric field distributions in cross-sections of $x-z$ plane at three transmission peaks of the composite nanocavities structure.

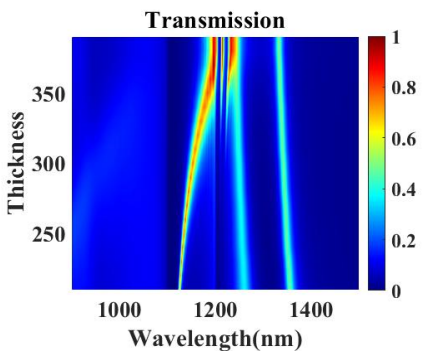

Figure 5. Simulated transmission spectra of the composite nanocavities metasurface with different $\mathrm{Au}$ film thicknesses $\mathrm{h}$.

The transmission spectrums of the composite nanocavities metasurface with different geometrical parameters S1 and S2 of vertical cavities are numerically investigated. As shown in Figure 6, transmission peak I remains almost unchanged, while peaks II and III are all shifted as S1 and S2 increase. S1 and S2 influence the coupling between plasmonic 
resonance in two vertical cavities, resulting in a change of the resonance wavelength of peaks II and III. Distance between different vertical cavities is utilized to control the spacing and position of the transmission peaks of the metasurface filter.
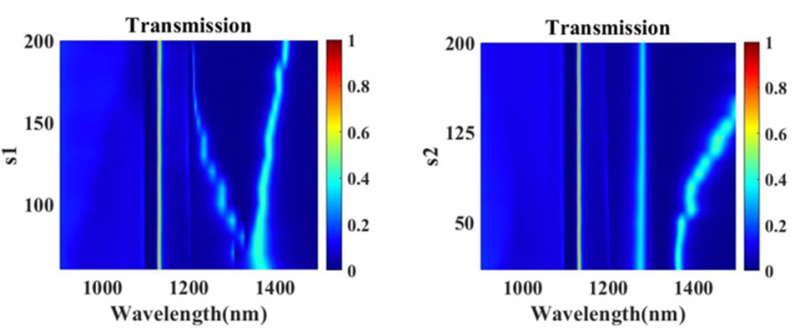

Figure 6. Simulated transmission spectra of the composite nanocavities metasurface with different S1 and S2.

The effect of lengths of the vertical nanocavities of the metasurface is also investigated. Transmission peak II is red-shifted with peaks I and III stay almost unchanged with L1 increasing from 0 to $400 \mathrm{~nm}$ in Figure 7. Similarly, an increase in L2 only causes the redshift of peak III. The length of a single vertical cavity only influences the EOT mode associated with itself and has no impact on other EOT peaks. Therefore, the EOT peaks II and III related to hybridization modes are able to be adjusted separately by lengths of the vertical nanocavities.
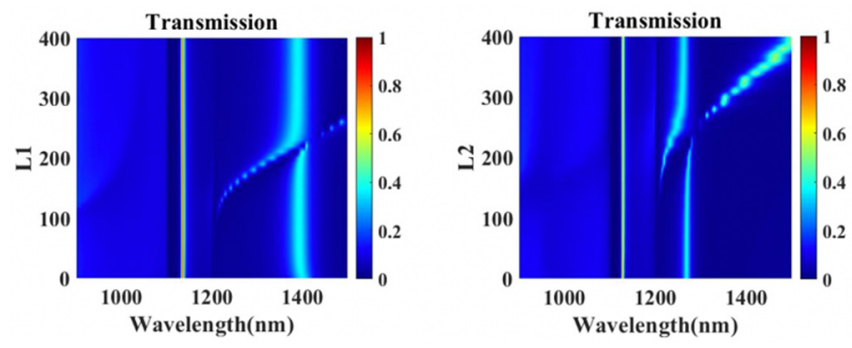

Figure 7. Simulated transmission spectra of the composite nanocavities metasurface with different lengths of vertical nanocavities L1 and L2.

The effect of periods of the composite nanocavities metasurface on the transmission spectrum is also numerically calculated by the FDTD solution. When lateral period Px increases from 1100 to $1300 \mathrm{~nm}$, peak I presents a slightly blue shift, and peaks II and III are red-shifted, as shown in Figure 8. With vertical period Py increasing from 1000 to $1250 \mathrm{~nm}$, peak I has an obvious red shift, while peaks II and III are almost unchanged. The interaction of vertical cavities in adjacent periods is mainly influenced by Px, while that of the horizontal cavity is sensitive to Py, resulting in peak I and peaks II/III modulated by Py and Px, respectively. The spectral positions of EOT peak I and peaks II/III are independently tuned by the periods of metasurface in different directions.

The spectra response of environmental refractive index of composite nanocavities metasurface is also simulated by FDTD. As the environment refractive index $\mathrm{n}_{\mathrm{e}}$ increases, the EOT peaks I, II, and III on transmission spectrum influenced by surface plasmon resonance are all red-shifted (shown in Figure 9a), and the refractive index sensitivity $\mathrm{S}(\delta \lambda / \delta \mathrm{n})$ are 1143,1245 , and $1360 \mathrm{~nm} / \mathrm{RIU}$ (shown in Figure $9 \mathrm{~b}$ ), respectively. The transmission peaks of the composite nanocavities metasurface have quite high refractive index sensitivity. As a near-infrared multiple-channel filter, which is sensitive to the surrounding environment, it offers a feasible way to realize an environment-refractive tunable filter and provides the potential to be the integration component in nano-sensing. 


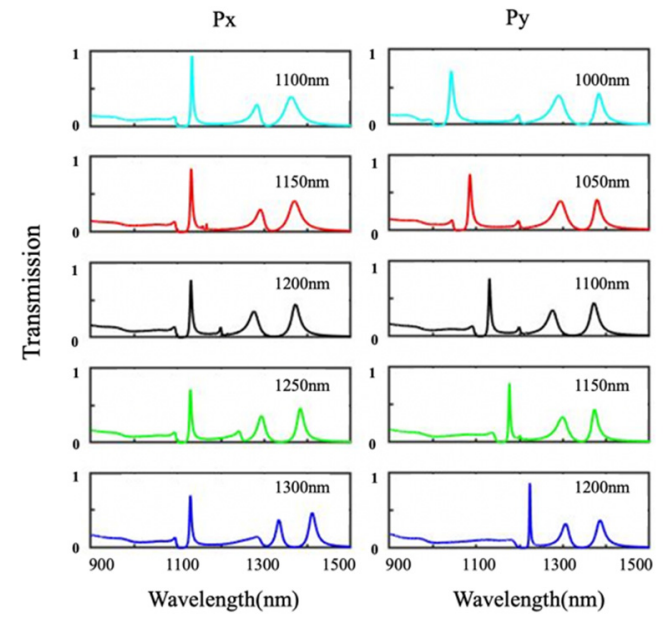

Figure 8. Simulated transmission spectra of the composite nanocavities metasurface with different periods Px and Py.
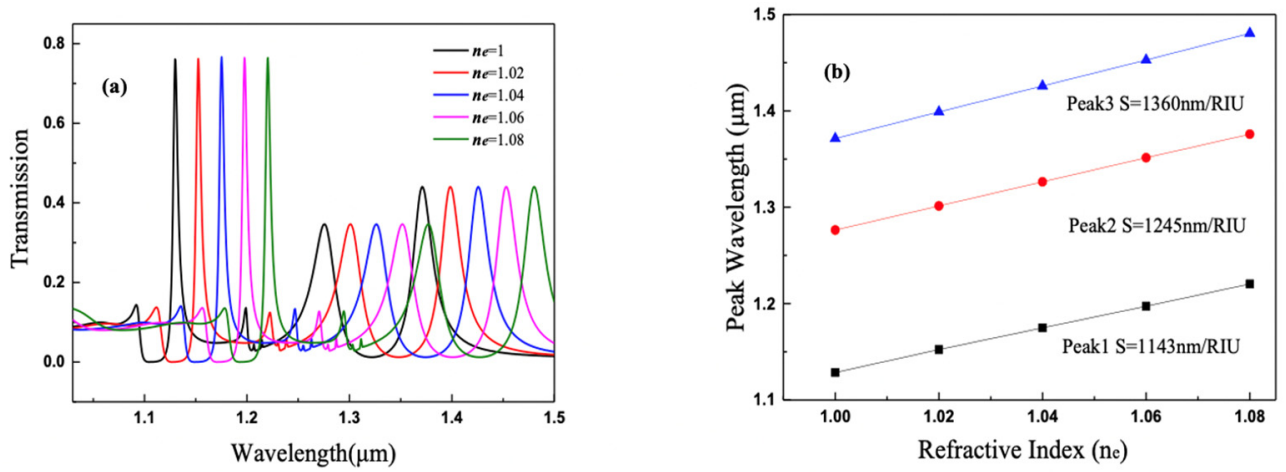

Figure 9. (a) Transmission spectra of composite cavities metasurface with different environmental refractive index $n_{e}$. (b) The wavelength of three transmission peaks versus the refractive index $n_{e}$.

The impact of incident angle on transmission spectrum is simulated as shown in Figure 10. Although transmission intensities of three peaks change simultaneously when the incident angle is varied, the wavelength positions do not shift. When the incident angle variation from the normal incidence is over \pm 10 degree, the impact on transmission intensity increased.

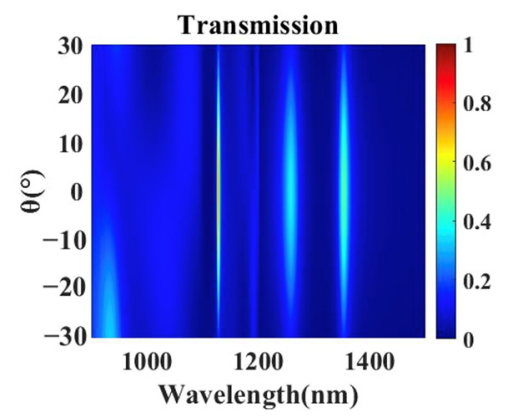

Figure 10. Simulated transmission spectra of the composite nanocavities metasurface with different incident angles $\theta$ ( $\theta$ represents incident angle, and $\theta=0$ degree represents normal incidence).

\subsection{Experimental Results}

A plasmonic infrared multiple-channel filter based on the composite nanocavities metasurface was fabricated. The $225 \mathrm{~nm}$-thick gold film was deposited on the $\mathrm{SiO}_{2}$ substrate by the Denton Electron Beam evaporator. The composite nanocavities metasurface was then fabricated on the gold film by Electron-beam lithography and Ion Beam Etching 
technique. The SEM image of the fabricated structure is shown in Figure 11. The geometrical parameters were measured in the SEM image: the length of the horizontal cavity is $\mathrm{L} 1=802 \mathrm{~nm}$, the lengths of vertical cavities are L2 $=204 \mathrm{~nm}$ and L3 $=301 \mathrm{~nm}$, the horizontal and vertical periods are $P x=1187 \mathrm{~nm}$ and $P y=1091 \mathrm{~nm}$, respectively. The size of the fabricated area is $1.2 \mathrm{~mm} \times 1.1 \mathrm{~mm}$. The transmission spectrum of the fabricated metasurface is detected by a Fourier-transform infrared spectrometer with a polarized source, which is shown as a red line in Figure 11. Transmission peaks at wavelengths $1128 \mathrm{~nm}, 1245 \mathrm{~nm}$, and $1362 \mathrm{~nm}$ of the measured spectrum exhibit good accordance in spectral tendency and positions with the simulation result (black line in Figure 11). However, the narrow FWHW and high transmittance of the metasurface in the simulation were not entirely presented in the experiment.

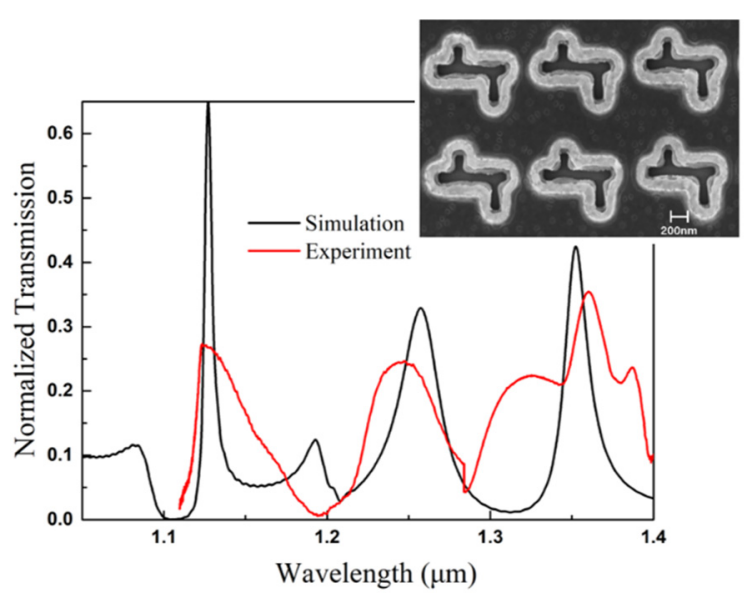

Figure 11. SEM images and the transmission spectrum of the fabricated composite nanocavities metasurface.

Several possible reasons of difference between simulation and experimental result are analyzed: imprecision in the EBL process leads to a loss of fidelity; the IBE process may cause damage on the Au surface; the dielectric parameter of Au in the FDTD simulation is set as Palik model, which has a minor difference with that of experiment [30]; the ohmic loss within the Au film, etc. Especially, the transmittance of the first peak in the simulation was not entirely presented in the experiment, for the narrow FWHW making it more susceptible to facing the experiment imperfection.

For future research, a dielectric parameter of $\mathrm{Au}$ in the simulation could be set from experimental determination rather than the Palik model to decrease the difference between simulation and experimental results. The fabrication process might be improved by using other alternative techniques such as FIB with implantation of Ga+ [31] to increase the geometrical accuracy of the fabricated metasurface.

The composite nanocavities metasurface with a brief single-layer fabrication process achieved the multi-peak EOT phenomenon in the near-infrared region, which implements the multi-frequency selective filtering at the nanoscale.

\section{Conclusions}

In conclusion, a plasmonic infrared multiple-channel filter based on a gold periodic composite nanocavities metasurface is numerically analyzed and experimentally demonstrated. The multi-peak extraordinary optical transmission phenomenon of the metasurface is generated from the coupling between different plasmonic modes in composite cavities, processing multiple-channel filtering at wavelengths $1126 \mathrm{~nm}, 1255 \mathrm{~nm}$, and $1350 \mathrm{~nm}$ in simulation. Surface plasmon resonances in composite cavities are affected by geometrical parameters of the cavities, periods of the metasurface, and environmental refractive index, which are utilized to tune frequency positions, spacing, and intensity of EOT peaks. The plasmonic composite nanocavities metasurface was fabricated by Electron-Beam Lithog- 
raphy and Ion Beam Etching technique. The experimentally measured spectrum of the fabricated metasurface by a Fourier-transform infrared spectrometer obtained three transmission peaks, which implemented multi-channel filtering at the nanoscale in the nearinfrared region. The multi-frequency selective ability and brief single-layer fabrication of the proposed plasmonic near-infrared filter are essential for future spatial applications in nano-optical systems such as biosensing and detecting.

Author Contributions: Software, J.Z., S.L. and C.S.; validation, J.Z., X.Y. and W.Y. formal analysis, X.Y. and J.D. (Jingxin Dong); data curation, J.Z. and J.D. (Jiacheng Duan); writing-original draft preparation, J.Z.; writing-review and editing, X.D.; funding acquisition, X.D. All authors have read and agreed to the published version of the manuscript.

Funding: This research was funded by the National Science Foundation of China (61927822).

Data Availability Statement: Data is contained within the article.

Acknowledgments: We thank the Center of Advanced Electronic Materials and Devices (AEMD) in Shanghai Jiao Tong University, where our nanostructure metasurface was fabricated.

Conflicts of Interest: The authors declare no conflict of interest.

\section{References}

1. Afzal, M.U.; Matekovits, L.; Esselle, K.P.; Lalbakhsh, A. Beam-Scanning Antenna Based on Near-Electric Field Phase Transformation and Refraction of Electromagnetic Wave Through Dielectric Structures. IEEE Access 2020, 8, 199242-199253. [CrossRef]

2. Das, P.; Kaushik, M.; Ali, L. Single-layer polarization-insensitive frequency selective surface for beam reconfigurability of monopole antennas. J. Electromagn. Waves Appl. 2020, 34, 86-102. [CrossRef]

3. Lalbakhsh, A.; Afzal, M.U.; Hayat, T.; Esselle, K.P.; Mandal, K. All-metal wideband metasurface for near-field transformation of medium-to-high gain electromagnetic sources. Sci. Rep. 2021, 11, 1-9. [CrossRef]

4. Wang, Y.; Chong, H.B.; Zhang, Z.; Zhao, Y. Large-area fabrication of complex nanohole arrays with highly tunable plasmonic properties. ACS Appl. Mater. Interfaces 2020, 12, 37435-37443. [CrossRef] [PubMed]

5. Suzuki, T.; Sekiya, M.; Sato, T.; Takebayashi, Y. Negative refractive index metamaterial with high transmission, low reflection, and low loss in the terahertz waveband. Opt. Express 2018, 26, 8314-8324. [CrossRef]

6. Wang, F.; Martinson, A.B.; Harutyunyan, H. Efficient nonlinear metasurface based on nonplanar plasmonic nanocavities. ACS Photonics 2017, 4, 1188-1894. [CrossRef]

7. Liu, L.; Wu, L.; Zhang, J.; Li, Z.; Zhang, B.; Luo, Y. Backward phase matching for second harmonic generation in negative-index conformal surface plasmonic metamaterials. Adv. Sci. 2018, 5, 1800661. [CrossRef] [PubMed]

8. Liang, Y.; Lu, M.; Chu, S.; Li, L.; Peng, W. Tunable plasmonic resonances in the hexagonal nanoarrays of annular aperture for biosensing. Plasmonics 2016, 11, 205-212. [CrossRef]

9. Zhu, J.; Lin, G.; Huang, Y.; Zhang, K.; Wu, M.; Wu, W.; Lu, P. Three-dimensional cavity-coupled metamaterials for plasmonic color and real-time colorimetric biosensors. Nanoscale 2020, 12, 4418-4425. [CrossRef] [PubMed]

10. Kazanskiy, N.L.; Butt, M.A.; Khoniana, S.N. Carbon dioxide gas sensor based on polyhexamethylene biguanide polymer deposited on silicon nano-cylinders metasurface. Sensors 2021, 21, 378. [CrossRef] [PubMed]

11. Lin, Y.S.; Dai, J.; Zeng, Z.; Yang, B.R. Metasurface color filters using aluminum and lithium niobate configurations. Nanoscale Res. Lett. 2020, 15, 1-8. [CrossRef]

12. Sabra, W.; Azzam, S.I.; Song, M.; Povolotskyi, M.; Aly, A.H.; Kildishev, A.V. Plasmonic metasurfaces for subtractive color filtering: Optimized nonlinear regression models. Opt. Letters 2018, 43, 4815-4818. [CrossRef] [PubMed]

13. Han, X.; Fan, Z.; Liu, Z.; Li, C.; Guo, L.J. Inverse design of metasurface optical filters using deep neural network with high degrees of freedom. InfoMat 2021, 3, 432-442. [CrossRef]

14. Si, G.; Zhao, Y.; Liu, H.; Teo, S.; Zhang, M.; Jun Huang, T.; Danner, A.J.; Teng, J. Annular aperture array based color filter. Appl. Phys. Lett. 2011, 99, 033105. [CrossRef]

15. Huang, T.J.; Yin, L.Z.; Shuang, Y.; Liu, J.Y.; Tan, Y.; Liu, P.K. Far-field subwavelength resolution imaging by spatial spectrum sampling. Phys. Rev. Appl. 2019, 12, 034046. [CrossRef]

16. Luo, M.; Zhou, Y.; Wu, S.; Chen, L. Wide-angle broadband absorber based on one-dimensional metasurface in the visible region. Appl. Phys. Express 2017, 10, 092601. [CrossRef]

17. Yannopapas, V. Periodic Arrays of Film-Coupled Cubic Nanoantennas as Tunable Plasmonic Metasurfaces. Photonics 2015, 2, 270-278. [CrossRef]

18. Koshelev, K.; Lepeshov, S.; Liu, M.; Bogdanov, A.; Kivshar, Y. Asymmetric metasurfaces with high-Q resonances governed by bound states in the continuum. Phys. Rev. Lett. 2018, 121, 193903. [CrossRef] 
19. Dhama, R.; Caligiuri, V.; Petti, L.; Rashed, A.R.; Rippa, M.; Lento, R.; Termine, R.; Caglayan, H.; De Luca, A. Extraordinary effects in quasi-periodic gold nanocavities: Enhanced transmission and polarization control of cavity modes. ACS Nano 2018, 12, 504-512. [CrossRef]

20. Ma, W.; Yu, S.; Zhao, T. Numerical study of an ultra-broadband perfect absorber from ultraviolet to near-infrared based on metal quadruple staircase structure. Opt. Commun. 2021, 493, 127037.

21. Sun, Z.; Zhao, J.; Zhu, B.; Jiang, T.; Feng, Y. Selective wave-transmitting electromagnetic absorber through composite metasurface. AIP Adv. 2017, 7, 115017. [CrossRef]

22. Gao, B.; Wang, Y.; Zhang, T.; Xu, Y.; He, A.; Dai, L.; Zhang, J. Nanoscale refractive index sensors with high figures of merit via optical slot antennas. ACS Nano 2019, 13, 9131-9138. [CrossRef] [PubMed]

23. Jiang, L.; Li, Y.; Wang, H.; Zhu, Z.; Yuan, Q.; Wang, J.; Qu, S. Composite metasurface merging frequency selective surface and coding sequences for electromagnetic transmission-diffusion. J. Phys. D Appl. Phys. 2021, 54, 235304. [CrossRef]

24. Sun, D.; Qi, L.; Liu, Z. Terahertz broadband filter and electromagnetically induced transparency structure with complementary metasurface. Results Phys. 2020, 16, 102887. [CrossRef]

25. Tung, B.S.; Khuyen, B.X.; Kim, Y.J.; Lam, V.D.; Kim, K.W.; Lee, Y. Polarization-independent, wide-incident-angle and dual-band perfect absorption, based on near-field coupling in a symmetric metamaterial. Sci. Rep. 2017, 7, 1-7. [CrossRef]

26. Shah, Y.D.; Grant, J.; Hao, D.; Kenney, M.; Pusino, V.; Cumming, D.R. Ultra-narrow line width polarization-insensitive filter using a symmetry-breaking selective plasmonic metasurface. ACS Photonics 2018, 5, 663-669. [CrossRef]

27. Zhang, J.; Dai, J.; Yang, J.; Hu, C.; Gao, B.; Xu, Y.; Zhang, J. Invertible plasmonic spin-Hall effect at nanoscale based on U-shaped optical slot nanoantenna. Nanotechnology 2019, 30, 345201. [CrossRef]

28. Hong, J.; Son, H.; Kim, C.; Mun, S.E.; Sung, J.; Lee, B. Absorptive metasurface color filters based on hyperbolic metamaterials for a CMOS image sensor. Optics Express 2021, 29, 3643-3658. [CrossRef]

29. Lalbakhsh, A.; Esselle, K.P. Directivity improvement of a Fabry-Perot cavity antenna by enhancing near field characteristic. In Proceedings of the 17th International Symposium on Antenna Technology and Applied Electromagnetics (ANTEM), Montreal, QC, Canada, 10-13 July 2016.

30. To, N.; Juodkazis, S.; Nishijima, Y. Detailed Experiment-Theory Comparison of Mid-Infrared Metasurface Perfect Absorbers. Micromachines 2020, 11, 409. [CrossRef]

31. Seniutinas, G.; Balčytis, A.; Reklaitis, I.; Chen, F.; Davis, J.; David, C.; Juodkazis, S. Tipping solutions: Emerging 3D nanofabrication/-imaging technologies. Nanophotonics 2017, 6, 923-941. [CrossRef] 